\title{
The Clinical Champions Programme: Growing local leaders to transform diabetes care
}

Authors: Florence Findlay-White, Beth Stout, Amy Rylance, Mhukti Perumal (all Diabetes UK)

\section{Background}

We are facing a diabetes crisis. An estimated $\mathbf{4 . 5}$ million people are living with diabetes in the UK. To safely manage their condition and live well, people with diabetes need support from their healthcare teams. But around $60 \%$ of people with Type 1 diabetes and $\mathbf{4 0} \%$ of people with Type 2 diabetes do not get the checks they need to avoid devastating complications such as heart disease, blindness and amputations.

The Clinical Champions programme recognises that clinicians are well-placed to transform diabetes services so that people with diabetes get the care they need to live well. As clinicians often don't have the leadership skills and confidence to drive service change, the Clinical Champions programme aims to give them the support to do this.

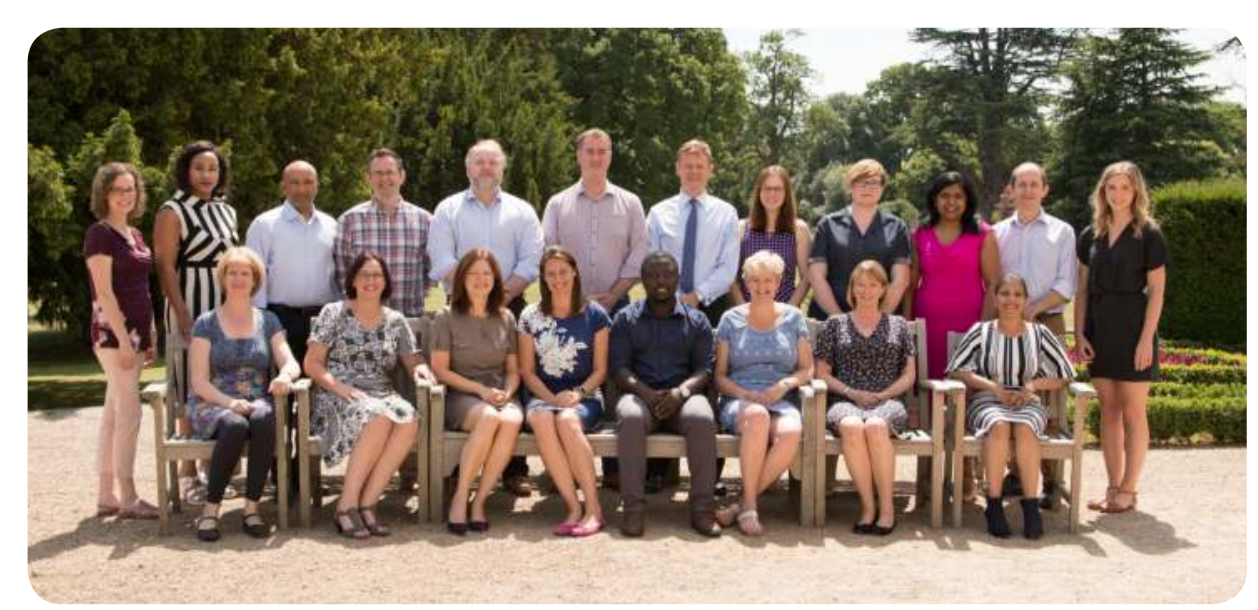

\section{(20) Aims}

The Clinical Champions programme has three main aims:

1 To develop clinicians' leadership capabilities enabling them to drive improvements in local diabetes care.

2 To develop a network of like-minded clinicians to share best practice, facilitating the spread of innovation across the UK.

3 To highlight the need for and raise the profile of clinical leadership.

\section{Method}

To establish the programme, we partnered with Novo Nordisk and Ashridge Business School.

Each year, we recruit 20 Clinical Champions from across diabetes care to the two-year programme.

They receive the following support:

- Leadership training at the world-renowned Ashridge Business School.

- Peer support through Action Learning Sets.

- Media coverage to raise their profile.

- Ongoing support from Diabetes UK to lead an improvement project.

\section{Results}

The programme has propelled 65 clinicians to become local leaders.

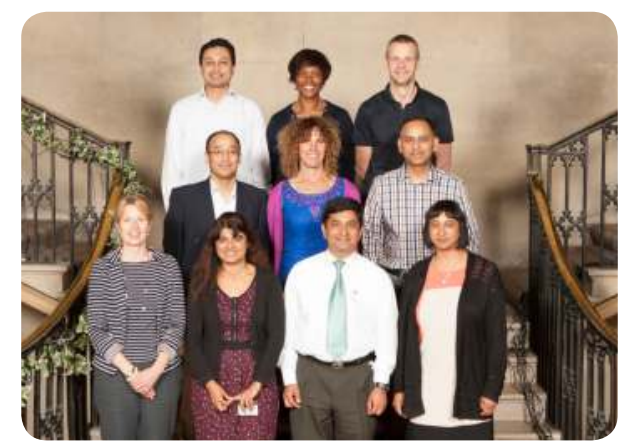

The improvement projects led by the Clinical Champions have transformed local care. Achievements include:

- Implementation of a Birmingham-wide formulary for diabetes medication, ensuring all patients are entitled to the same treatment no matter where they live in the area.

- Delivery of an accessible diabetes training programme to over 1,000 inpatient and community nurses. This contributed to a reduction in insulin errors from $24.5 \%$ in 2015 to $13.3 \%$ in 2016.

- Recruitment of the first diabetes schools educator in Wales to tackle the issue of schools giving inadequate care to children with diabetes.

- More than 18,000 people across Scotland using technology to monitor their diabetes in clinic.

As a result of the success of the programme, we have secured funding from Novo Nordisk to recruit another 20 Clinical

Champions in 2018.

\section{Discussion}

The programme has demonstrated that giving clinicians the opportunity to develop their leadership skills helps them improve local diabetes services. Clinical Champions have told us:
"The Clinical Champions programme has benefitted me immensely and has helped me understand the potential for change."

"The programme supports people in numerous different ways to be able to achieve things that they never thought they could in terms of diabetes care improvement."
"Being a Clinical Champion and having the backing of Diabetes UK has helped to raise awareness of diabetes in my local area and put it on my trust's agenda." 